The following text is a post-print (ie final draft post-refereeing) version of the article which differs from the publisher's version.

To cite this article use the following citation:

Lorenzi R, Zullino A, Prosperi L, Paleari A

"Visible-light excited red-emitting vacancies at carbon interstitials as indicators of irradiated and annealed Type la diamonds"

DIAMOND \& RELATED MATERIALS, Vol. 90, p. 188-193, 2018

doi: 10.1016/j.diamond.2018.10.014

Publisher's version of the article can be found at the following site:

https://www.sciencedirect.com/science/article/pii/S0925963518303753 


\title{
Visible-light excited red-emitting vacancies at carbon interstitials as indicators of irradiated and annealed Type Ia diamonds
}

\author{
Roberto Lorenzi ${ }^{1} *$, Andrea Zullino ${ }^{2}$, Loredana Prosperi ${ }^{2}$, Alberto Paleari ${ }^{1}$ \\ ${ }^{1}$ Department of Materials Science, University of Milano - Bicocca, via Cozzi 55, 20125 Milano, \\ Italy \\ ${ }^{2}$ Istituto Gemmologico Italiano (IGI), piazza San Sepolcro 1, 20123 Milano, Italy
}

During the last decades many studies have been carried out to investigate how point defects and aggregates respond and evolve in natural Type Ia diamonds as a result of treatments, and a number of underlying mechanisms have been identified and interpreted. However, the analysis of radiation-induced creation/ionization of defects, as well as their migration and aggregation in secondary defect structures, often requires experimental approaches which can hardly constitute a simple-to-use diagnostic tool for the identification of artificially treated diamonds. Here we disclose a novel simple indicator of artificial exposure of Type Ia diamonds to ionizing radiations and subsequent annealing. This indicator consists in narrow photoluminescence lines in the red region, between 681 and $725 \mathrm{~nm}$, we recently found to result from vacancies trapped by interstitial carbon aggregates and platelets. Our results demonstrate that interstitial structures become sites of vacancy trapping - by thermal migration of radiation-induced vacancies - only when diamond undergoes treatments. We give the rigorous validation of the new spectroscopic probe of artificial treatments analysing photoluminescence and infrared absorption spectra of well-known H1b and H1c centres in a hundred samples. Importantly, the method is based on emission lines which do not require neither high photon-energy excitation nor cryogenic temperatures.

keywords: diamond crystal, natural diamond, absorption, optical emission, radiation induced effects, defect characterization, optical properties characterization, vibrational properties characterization, defects, electronic states, optical properties, vibrational properties, irradiation and annealing.

\footnotetext{
*Corresponding author: Roberto Lorenzi, Tel: +39 0264485169, e-mail: roberto.lorenzi@unimib.it
} 


\section{Introduction}

The mechanisms of formation and migration of carbon vacancies in diamond as a function of synthesis and post-synthesis processes determine many of the light emission and charge transport properties which are of topical interest for several technological applications of this material [1-7]. At the same time, defect-related mechanisms are involved in the artificial treatments which have been employed for many years to modify the colour of natural diamonds [8-13]. For this reason, a number of gemmological analysis techniques have been implemented on the basis of defect spectroscopy, with the aim of detecting whether a diamond has been artificially treated, such as by neutron or electron irradiation and/or by annealing processes. In fact, defectiveness on atomic scale turns out to be different in natural untreated diamonds compared to natural diamonds exposed to ionizing radiation and thermal heating, regardless of the colour similarity or even lack of difference between the two types of gemstones by visual inspection.

Along this line of investigation, many studies analyzed the varieties of defects which are induced by ionizing irradiation and annealing processes in Type-I and Type-II diamonds [10]. These studies showed that the vacancy production rates are related to the preexisting diamond defects and that these systems can be modified by treatments, such as high pressure high temperature (HPHT) processes, and even only by simple heat treatments at sufficiently high temperature. Depending on the concentration of impurities - mainly boron, nitrogen, and related aggregates - different defects can form in the diamond lattice, such as the well known GR1, ND1, H1b, H1c, H2, H3, H4, 5RL, 3H, N-V and $595 \mathrm{~nm}$ centres [14]. Furthermore, all these optical centres can occur in very different concentrations and show different creation rates depending on the diamond type and the conditions of irradiation and thermal treatment.

Several studies investigated the high temperature annealing behaviour of optical centres in irradiated diamonds, specifically the GR1 centre absorbing at $741 \mathrm{~nm}$, the H3 centre at $503 \mathrm{~nm}$ and the zero-phonon line at $595 \mathrm{~nm}(2.086 \mathrm{eV})$, concluding that the absence of the 595-nm line does not unambiguously categorise a diamond as untreated and that further clues come from the relative strengths of the $\mathrm{H} 3$ and the $\mathrm{H} 4$ (at $496 \mathrm{~nm}$ ) absorption bands [8]. Indeed, the temperature dependence of the $595 \mathrm{~nm}$ between 700 and $1000{ }^{\circ} \mathrm{C}$ in irradiated Type-Ia diamonds highlights some kind of relationship with the defects responsible for the H1b (2024 nm) and H1c (1934 nm) zero-phonon absorption lines in the infrared (IR) spectrum [9], showing that H1b and H1c centres are formed when all, or part, of the $2.086 \mathrm{eV}$ centres are trapped at the A and $\mathrm{B}$ aggregates of nitrogen during the annealing process. Therefore, although $\mathrm{A}$ and $\mathrm{B}$ nitrogen aggregates are present in natural diamonds, the defect complexes arising from vacancies migration to A and B sites appear to be peculiar of treated samples [13, 15-17]. In fact, the presence of H1b and H1c centres is an 
extremely rare event in natural diamonds, since the natural annealing in the Earth is expected to completely destroy the defects after formation [15]. Notable exceptions are some purple diamonds from Siberia [18], and a 14 carats yellow diamond of unknown origin studied by Collins [19, 20], which was cut before 1950 and, for this reason, presumably neither irradiated nor heated.

The processes that lead to the formation of GR1, H1b, H1c, H2, and H3 are also involved in the treatments which can directly influence the visual colour of the gemstone. Several works in the literature are indeed devoted to the colour origin of diamonds and refer to electron or ion irradiation combined with annealing and to heat treatment under high-pressure and high-temperature (HPHT) as common treatment techniques [11]. In this regard, many studies showed that photoluminescence (PL) analysis with laser excitation at $514 \mathrm{~nm}$ or $488 \mathrm{~nm}$ is an effective tool to detect the HPHT process $[11,21]$, as well as absorption spectroscopy in the visible and IR spectrum. Laser-induced PL spectrocopy was in fact applied to identify treated diamonds, finding various lines in the PL spectra on type $\mathrm{IaAB}$ diamond, whose intensity turns out to depend on the dose and type of radiation, and apparently on the HPHT treatment conditions [12]. From all the studies on defects in diamond, one concludes that numerous clues to identifications can be detected by analysing diamonds with a wide range of spectroscopic techniques, and that the combination of different spectroscopic methods, including IR, optical absorption and PL spesctroscopy, could be successufully applied.

In this framework, we have recently found the spectroscopic signature of previously unknown carbon vacancy variants interacting with interstitial carbon aggregates in natural Type Ia diamonds, consisting in PL lines $\left(\mathrm{D}_{\mathrm{b}}\right.$ and $\mathrm{D}_{\mathrm{a}}$ doublets at low temperature, which become three lines at 681, 705 , and $725 \mathrm{~nm}$ at room temperature) in the red spectral region [22]. These signals have been previuosly reported in literature, even though their exact origin was quite unclear and based on empirical observation in treated diamonds without robust experimental evidences of the local atomic arrangement responsible for the observed spectral lines [14, 23]. In particular, emission line at $681 \mathrm{~nm}$ has been observed by Osvet [24, 25], Nisida [26], and Hainschwang [16] and coworkers in neutron and electron irradiated diamonds followed by annealing at $\mathrm{T}>700^{\circ} \mathrm{C}$. Emission at 681 $\mathrm{nm}$ has been registered also in natural Type I diamonds [25], but only at $\mathrm{T}<80 \mathrm{~K}$. The pair of lines at $705 \mathrm{~nm}$ and $725 \mathrm{~nm}$ has never been reported as a doublet, but the single emission lines have been reported by Osvet [22, 24], Vins [27], and coworker in neutron irradiated and annealed Type I diamonds, but then again in spectra taken at low temperatures.

In the present work, from a set of a hundred Type Ia diamonds, we unveil and statistically validate a strict and unprecedented relationship between the occurrence of the $\mathrm{Da}$ and $\mathrm{Db}$ lines as light-emitting vacancy variants and the spectroscopic evidences of H1b and H1c which are typically 
generated by irradiation and heating treatments. We give evidences for the first time that carbon interstitial aggregates and platelets can be sites of vacancy condensation in the diamond structure undergoing thermally activated migration of radiation-induced vacancies. Importantly, as a consequence of our analysis, the spectroscopic signature of such light-emitting vacancy variants can become a novel easy-to-use diagnostic probe of artificial treatments in Type Ia natural diamonds.

\section{Materials and methods}

The experimental investigation involves a set of a hundred Type Ia natural diamonds, few $\mathrm{mm}^{3}$ in size on average. The sample set comprises 50 colour enhanced natural diamonds and 50 untreated natural diamonds, and all are yellow diamonds. The samples are of unknown provenance and they are all of gemmological and commercial interest. Samples underwent standard gemmological analysis, including visual inspection, short- and longwave UV luminescence, UV/Vis and IR absorption, and photoluminescence at liquid nitrogen excited at $488 \mathrm{~nm}$. The artificial treatments of treated diamonds are unknown. However, the results of gemmological tests showed the presence of $\mathrm{H} 1 \mathrm{~b}$ and H1c centres, compatible with irradiation with high energy particles followed by thermal treatments at $\mathrm{T}>700^{\circ} \mathrm{C}$. Tests on natural samples did not revealed any sign ascribable to artificial treatments.

IR measurements were carried out at room temperature by means of a Fourier-Transform spectrometer (Frontier by Perkin Elmer) in the range $800-6000 \mathrm{~cm}^{-1}$ with a spectral resolution of 4 $\mathrm{cm}^{-1}$, spectra were collected with a diffuse reflectance (DRIFT) accessory. PL measurements were carried out at room temperature using a $\mathrm{HeNe}$ laser at $633 \mathrm{~nm}$ as excitation source and with a density power of the beam of the order of $0.1 \mathrm{~W} / \mathrm{mm}^{2}$. The light signal was collected by a chargecoupled-device after dispersing the signal by a polychromator in the range 655-900 nm with a final resolution of about $0.2 \mathrm{~nm}$. Spectra were collected with a micro-spectrophotometer LabRAM HR (Horiba - Jobin Yvon) working in backscattering configuration and using a 20x objective. 

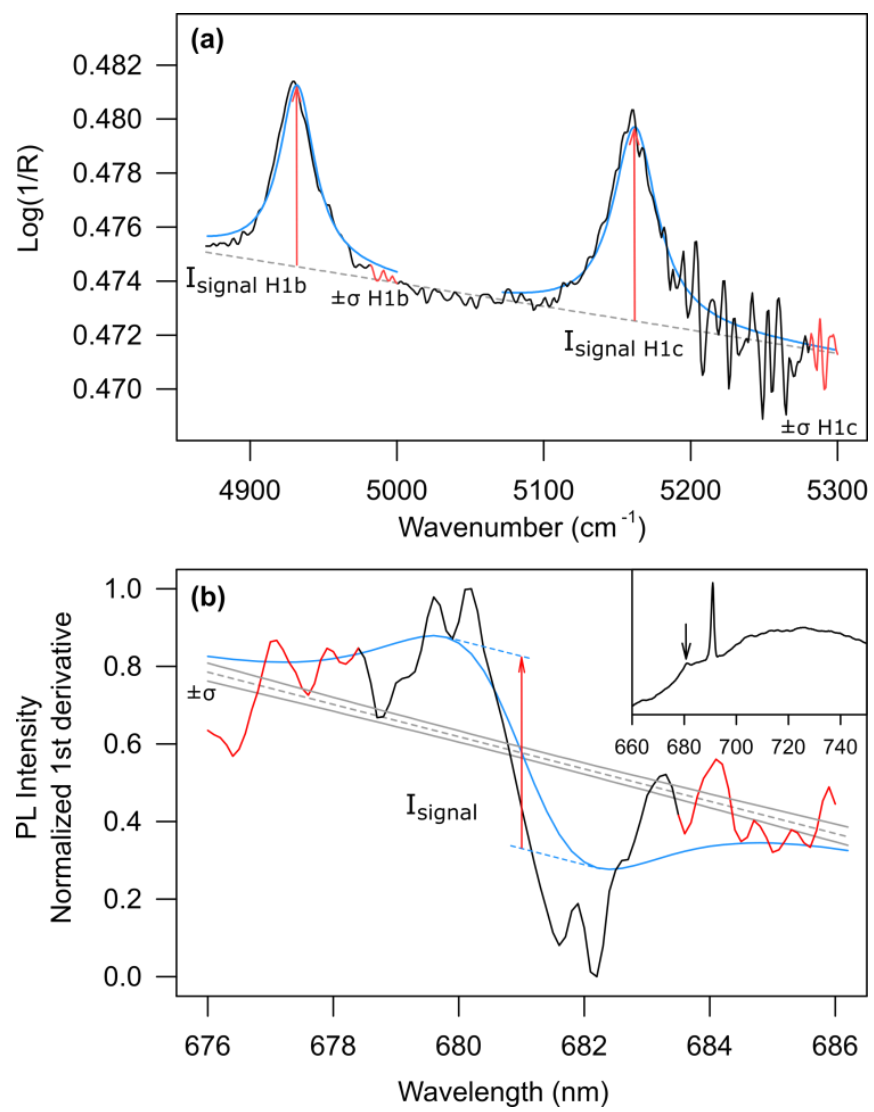

Fig 1.: Schematic representation of the procedure used for noise-to-signal ratio evaluation for (a) DRIFT measurements and (b) PL measurements. In the inset: raw PL spectrum used in this example, black arrow indicates the peak analysed in first derivative.

Raw spectra were further manipulated for quantitative comparison between different samples, as schematically depicted in Fig. 1. In particular, IR spectra in $\log (1 / R)$ scale, where $R$ is the measured diffuse reflectance, were normalized for the intensity at $3640 \mathrm{~cm}^{-1}$ which corresponds to intrinsic vibrational modes in the three-phonon region [28]. The values of peak heights at $4932 \mathrm{~cm}^{-1}$ and $5162 \mathrm{~cm}^{-1}$ (red arrows in Fig. 1a), corresponding to $\mathrm{H} 1 \mathrm{~b}$ and $\mathrm{H} 1 \mathrm{c}$ centres, respectively, obtained after baseline subtraction and fitting with Lorentzian functions (blue lines in Fig. 1a), were divided by a factor $\sigma_{I R}$. The latter represents the standard deviation of scattered data in a region without any signal (red parts of the spectrum in Fig. 1a) and close to the peak of interest, specifically at 4880$5000 \mathrm{~cm}^{-1}$ and $5280-5300 \mathrm{~cm}^{-1}$, for $\mathrm{H} 1 \mathrm{~b}$ and $\mathrm{H} 1 \mathrm{c}$ centres, respectively. Finally, the two signal-tonoise ratios were summed.

For quantitative comparison of PL data we used a slightly different method by analysing the signal first derivative. This approach overcomes problems related to the presence of very different band shapes in correspondence of the peaks of interest that would necessitate the use of non-trivial and sample-dependent baseline corrections. Instead, by using first derivative spectroscopy the peak is superimposed to local bands that can be treated as linear baselines. As in the case of IR quantitative comparison, signal is fitted with a first derivative Lorentzian peak (blue solid lines in 
Fig 1b) and its amplitude (red arrow in Fig. 1b) is divided by a factor proportional to noise. Noise has been calculated starting from linear regression of points lying $2 \mathrm{~nm}$ before and after the expected peak position (red parts of the spectrum in Fig. 1b), then taking the value of single prediction bands at peak maximum with a confidence level of 1 standard deviation (solid grey lines in Fig. 1b). In other words, the noise value - calculated from data dispersion in a peak-free region gives an estimation of the probability of $68 \%$ to find a point when no signal contributes in the considered spectral region. This procedure has been applied separately to each spectral region related to the peaks under study, the $\mathrm{Db}, \mathrm{Da}_{1}$, and $\mathrm{Da}_{2}$ falling at $681,705 \mathrm{~nm}$ and $725 \mathrm{~nm}$, respectively.

\section{Results and discussion}

\subsection{IR and PL variety within the sample set}

The statistical validation of PL signals as indicators of diamond treatment relies on a large set of samples all of natural origin. Thus the quantity and type of optically active defects are determined by the geological processes during gem formation and, for treated samples, by the artificial treatments. For this reason, the set presents a great variety of IR and PL spectra. In Fig. 2 we report five spectra of both natural and treated samples representative of the different spectroscopic behaviours found in our statistical validation. 

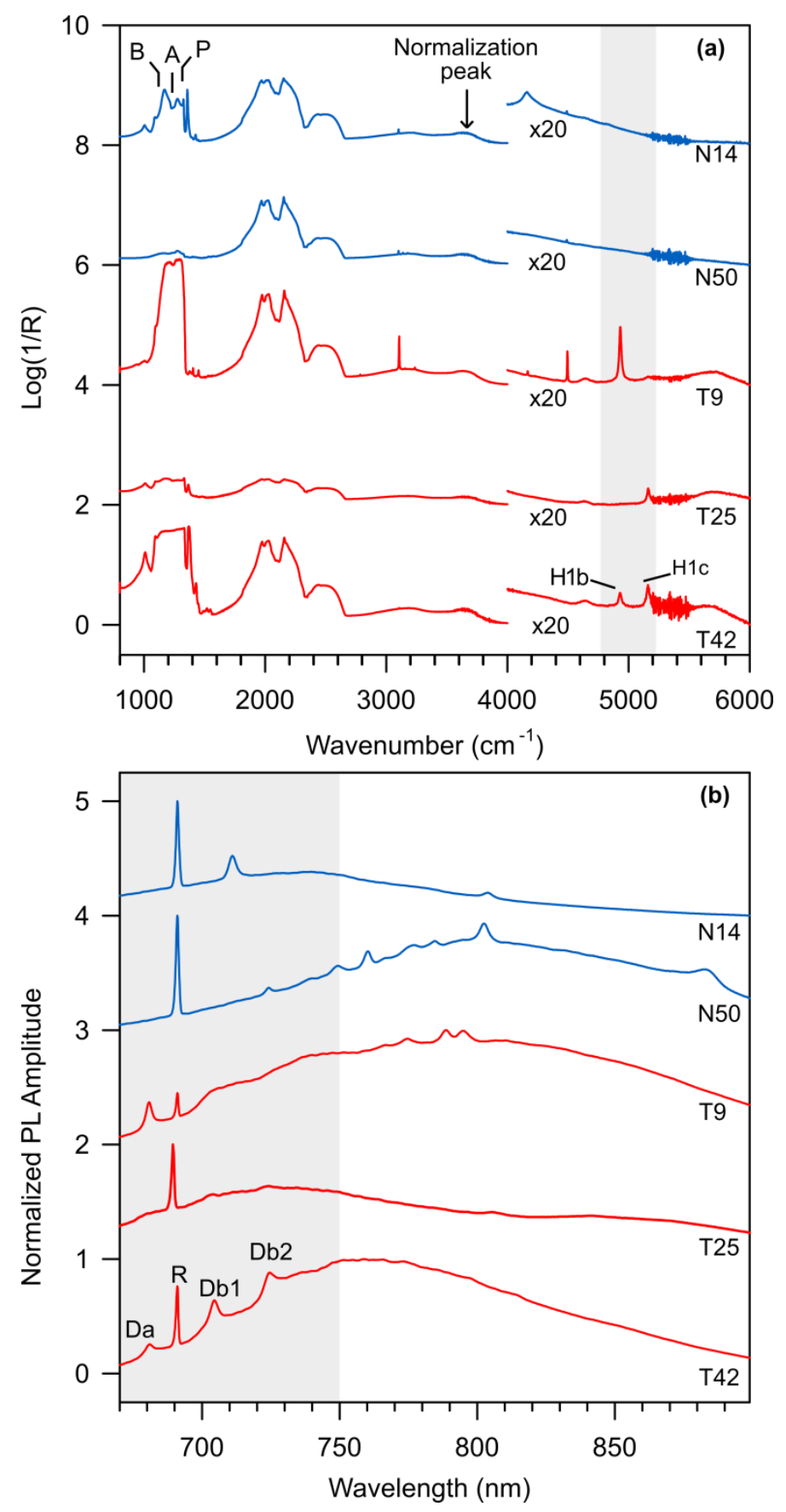

Fig. 2: (a) IR and (b) normalized PL spectra representative of the different spectroscopic behaviours found in our statistical survey. Spectra of natural and treated samples are labelled with a $\mathrm{N}$ and a $\mathrm{T}$, respectively, for numbers after letter refer to next Section. In IR spectra, normalization peak is indicated by an arrow, signals marked with A, B, and P, refer to A and B aggregates, and platelets, respectively. In PL spectra the signal marked as R refers to the Raman mode. Spectra in (a) and (b) are vertically shifted for clarity. Grey regions indicate the spectral bands taken into consideration in the statistical validation of this work.

The IR spectral features we find in the set of Type Ia diamonds are summarized by the spectra in Fig. 2a. Intense structures dominate the range between 1000 and $1500 \mathrm{~cm}^{-1}$ from spectral contributions due to nitrogen aggregates $\mathrm{A}$ and $\mathrm{B}$ [8], and the possible occurrence of interstitial carbon platelets [29]. At 1210 and $1282 \mathrm{~cm}^{-1}$ spectra show bands caused by A aggregates - i.e. 
defect complexes describable as $\mathrm{N}=\mathrm{N}$ pairs substituting for pairs of carbon atoms - while at 1170 and $1331 \mathrm{~cm}^{-1}$ the features of $\mathrm{B}$ aggregates, consisting of four nitrogen atoms substituting for carbon around a vacancy site. Isolated substitutional $\mathrm{N}$ atoms in $\mathrm{C}$ defects, if any, are instead expected to give bands at 1344 and $1110 \mathrm{~cm}^{-1}$, not observed in our set of Type Ia diamonds. At higher energy, at 1360-1370 $\mathrm{cm}^{-1}$, we register the phonon mode of interstitial carbon platelets. The amplified region above $4000 \mathrm{~cm}^{-1}$ is the spectral region in which we find the electronic transitions $\mathrm{H} 1 \mathrm{~b}$ and $\mathrm{H} 1 \mathrm{c}$ at 4932 and $5162 \mathrm{~cm}^{-1}$, respectively, which constitute the signature of irradiation and heating treatment of Type Ia diamonds, more specifically the treatment-induced evolution of A and $\mathrm{B}$ aggregates $[8,9,14]$. A first inspection of the reported spectra in the one-phonon region below $1500 \mathrm{~cm}^{-1}$ shows that all samples present $\mathrm{A}$ and $\mathrm{B}$ aggregates and the platelet peak, confirming they belong to Type Ia natural diamond species. The near-IR spectral region above $4000 \mathrm{~cm}^{-1}$ instead shows two quite distinct typologies of samples. The first one has intense H1b and H1c peaks (red lines in Fig. 2a), and indicates systems which underwent to artificial treatments of irradiation and heating. In the second one, the H1b and H1c peaks are absent (blue lines in Fig. 2a). This second set is in fact composed by certified samples of untreated natural Type Ia diamonds.

The heterogeneity of PL spectra in the investigated sample set of natural and treated diamonds is very rich regarding number, shape, and relative intensity of peaks and bands. The selected spectra reported in Fig. 2b show the most representative cases we have found in our experiments. All spectra have a common sharp peak at $691 \mathrm{~nm}$, intrinsic of the system under study, which corresponds to the Raman mode of diamond and falling $1332 \mathrm{~cm}^{-1}$ aside the excitation laser line at $633 \mathrm{~nm}$. Other broad bands may be present in the spectra in different spectral regions. Finally, superimposed to these broad features, there are a plethora of other peaks with intensities and sharpness comparable to the Raman peak. Within all the variety of peak shapes and positions some common features can be found. In particular, in all treated samples (red lines in Fig. 2b) three peaks are detected at 681,705 , and 725 . These three peaks at room temperature are what results from two doublets of lines we have recently studied down to $77 \mathrm{~K}$, i.e. the $\mathrm{D}_{\mathrm{a}}$ doublet with lines at 662 (undetectable at room temperature) and $681 \mathrm{~nm}$, and the $\mathrm{D}_{\mathrm{b}}$ doublet with lines at 705 and $725 \mathrm{~nm}$ [22]. In agreement with the attribution to two distinct doublets of lines - the $681 \mathrm{~nm}$ line to the $D_{a}$ doublet, and the 705, $725 \mathrm{~nm}$ lines to the $\mathrm{D}_{\mathrm{b}}$ doublet [22] - the peaks at 705 and $725 \mathrm{~nm}$ are always found together (see sample T42 and T25 in Fig. 2b), whereas the $681 \mathrm{~nm}$ line can occur without the two lines at longer wavelength (see sample T9 in Fig. 2b), and vice versa (see sample T25 in Fig. 2b). In addition, the great variety of PL signals that can be found in samples of natural origin gives rise to a large number of peaks including some cases in which at least one of the two lines of the doublet can be found alone (see sample N50 in Fig. 2b). From Fig. 2b, we also see that the two $D_{b}$ 
lines observed in treated samples have comparable intensity, and their intensity ratio does not show any sample dependence, at least within the experimental uncertainty, as expected from features belonging to electronic transitions of a single type of site. This result is even more stringent if compared with the broad luminescence background which is instead largely sample dependent. Transition energies and low temperature line splitting of the two doublets in treated samples match the energy structure expected from vacancies at interstitial aggregates [22]. The attribution to vacancy variants at interstitial structures is also suggested by the lack of D doublets in diamonds without detectable evidence of interstitial platelets (specifically, the infrared mode at 1360-1370 $\mathrm{cm}^{-1}$ ). In fact, several features of the sideband structures in the $\mathrm{D}_{\mathrm{b}}$ doublet (number of peaks, energy separation, and spectral linewidth) turn out to be related to the spectral position and bandwidth of the platelet IR mode, and to the expected motion of the interstitials through different equivalent positions and the related effects on the energy structure of the nearby vacancy [22]. It is worth to note that the occurrence of the $\mathrm{D}_{\mathrm{a}}$ and/or $\mathrm{D}_{\mathrm{b}}$ doublets was found in platelet-containing Type Ia diamonds with clear evidences of irradiation and heating treatment [22], as confirmed by the presence of the narrow absorption peaks $\mathrm{H} 1 \mathrm{~b}$ and $\mathrm{H} 1 \mathrm{c}$ at 4932 and $5162 \mathrm{~cm}^{-1}$, respectively [9]. This fact suggests that the vacancy variants at interstitial aggregates might result from irradiationinduced generation and thermally-activated migration of vacancies towards interstitial structures, such as platelets and other extended interstitial defects. To address this point, we collected PL spectra excited at $633 \mathrm{~nm}$ on Type Ia diamonds, with the aim of verifying the possible relationship between the three room temperature lines of the D doublets at 681, 705, and $725 \mathrm{~nm}$ and the IR signature of treated Type Ia diamonds.

\subsection{Analysis of the relationship between IR and PL features}

As a result of the points highlighted in the previous section, data suggest that the D lines are strongly related to the $\mathrm{H} 1$ peaks and the responsible defects are formed with comparable efficiency by irradiation and thermal treatment. This relationship also suggests that the atomic origin of these defects is the same. The widely accepted interpretation on the origin of H1b and H1c centres is the trapping of a nitrogen-vacancy pair defect trapped on the A- and B-aggregates of nitrogen, respectively [27]. In fact, irradiation causes the formation of the nitrogen-vacancy pair and the thermal treatments enable the migration of such defects to the nitrogen aggregates. In this scenario, data do not only show to be consistent with a model of vacancy variants driven by treatmentinduced mechanisms - including migration to interstitial platelet and similar aggregates - but also suggest that $\mathrm{D}$ lines can be used as a diagnostic system to distinguish treated from untreated natural 
Type I diamonds, provided that some caution is used so as to differentiate between independent generation of $\mathrm{D}_{\mathrm{b}}$ lines and other contributions from unknown defects at similar wavelength.

Following these suggestions, we can proceed by separately evaluating the intensity of the lines at $681 \mathrm{~nm}, 705$, and $725 \mathrm{~nm}$ in the whole sample set, and distinguishing between certified untreated diamonds and all other samples. All signal intensities from PL spectra have been evaluated by using the first derivative spectra as described in Section 2 (see Fig. 1b). The results of the analysis are reported in Fig. 3 which reports the signal-to-noise ratio for the lines at $681 \mathrm{~nm}$, 705, and $725 \mathrm{~nm}$ both for all treated samples (Fig. 3a) and all the untreated diamonds (Fig. 3b).
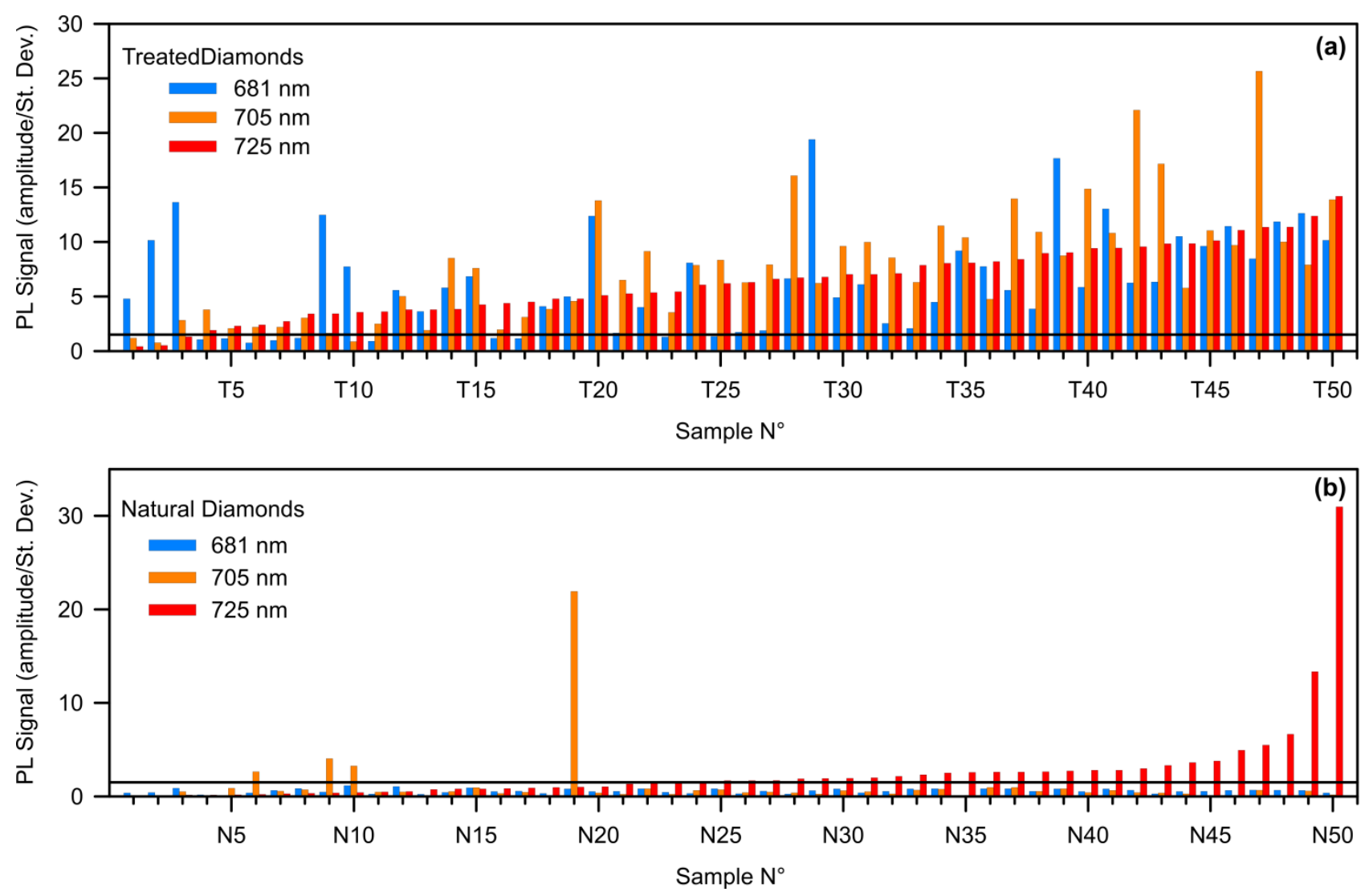

Fig. 3: Histogram of PL intensities at 681, 705 and $725 \mathrm{~nm}$, analysed as described in previous sections, in the set of treated (a) and control set of certified untreated samples (b). Samples are ordered and labelled according to increasing intensity of $725 \mathrm{~nm}$.

Looking at Fig. 3a, the detection of the $\mathrm{D}_{\mathrm{a}}$ line at $681 \mathrm{~nm}$ and/or both $\mathrm{D}_{\mathrm{b}}$ lines at 705 and 725 $\mathrm{nm}$ is a common feature of treated natural Type Ia diamonds within the relevant intensity threshold of 2 times the noise. Instead, looking at Fig. 3b, we notice that the emission at 681 does not reach an intensity higher than 2 times the standard deviation of the background of noise fluctuations throughout the whole set of natural untreated diamonds. Furthermore, in the same control set of untreated samples, there is no evidence of $D_{b}$ doublet. In fact, even though we detect some spectral structures at either 705 (sample N6, N9, N10, and N19) or $725 \mathrm{~nm}$ (sample from N32 to N50), in no 
sample we concomitantly register both lines with intensity higher than 2 times the noise standard deviation and with comparable intensity each other. Therefore, the lack of a significant peak at 681 $\mathrm{nm}$ and of a doublet of peaks at 705 and $725 \mathrm{~nm}$ with comparable significant intensity appears as a characteristic feature of untreated natural Type Ia diamonds.

We can get a more quantitative approach of a relationship between the infrared fingerprints of interstitial aggregate variant of vacancies and the possible effects on PL signals induced by irradiation and heating by comparing the integrated intensity of the H1b and H1c IR absorption transitions and that of D doublet lines. For this purpose, we have chosen as unique indicator of D lines intensity the maximum significant value (MSV). The latter is evaluated in two steps: 1) taking the smallest value between Db1 and Db2 intensities and 2) taking the largest value between the intensity found in the previous step and the Da peak. In such a way, for treated samples, we can have the following three cases: a) if only the Da line is present then the MSV corresponds to the Da intensity, b) if only the Db doublet is present then the MSV corresponds to the intensity of the lowest $\mathrm{Db}$ peak, or c) if both the $\mathrm{Da}$ line and the $\mathrm{Db}$ doublet are present then the MSV corresponds to either the $\mathrm{Da}$ intensity or the minimum intensity between the $\mathrm{Db} 1$ and $\mathrm{Db} 2$. For certified natural samples we used the same numerical procedure, but for all samples the MSV is automatically less than 2. Maximum significant values for all samples are reported in Fig. 4, together with the values of the sum of the H1b and H1c IR signal-to-noise ratios.

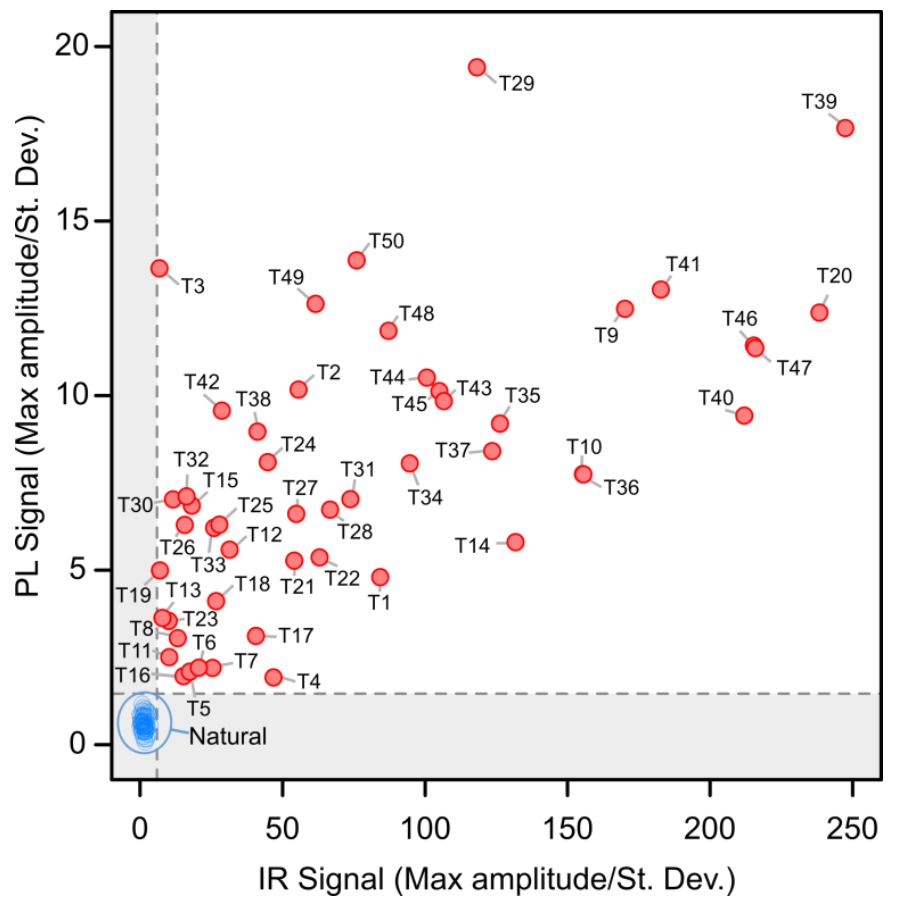

Fig. 4: Intensity of the maximum significant value (as described in the text) of $\mathrm{D}$ emission lines vs. the integrated intensity of the H1b and H1c IR peaks at 4932 and $5162 \mathrm{~cm}-1$, in all samples within the investigated Type Ia diamonds, comprising certified untreated samples (blue empty circles) and treated samples (red filled circles), grey regions are guide to the eyes for cut-off values of treatments discrimination. 
There are some relevant facts emerging from the data in Fig. 4. First, we notice that the experimental values approximate a linear relationship (except, importantly, for few points which we consider below) as expected if the D lines are due to vacancies generated and mobilized by mechanisms which are activated or favoured by the irradiation and heating treatments also responsible for $\mathrm{H} 1 \mathrm{~b}$ and $\mathrm{H} 1 \mathrm{c}$ centres. Second, all untreated natural samples (blue empty circles in Fig. 4) give values close to the origin of the axes. This fact strongly suggests that the conditions for the formation of detectable D lines and those for generating observable H1 peaks are equally unlike in nature. Third, values significantly deviating from the linear relationship - with weak H1 peaks and intense D lines (in particular samples T3 and T29 in Fig. 4) - are found in samples with the $D_{a}$ line at $681 \mathrm{~nm}$ accompanied by weak $\mathrm{D}_{\mathrm{b}}$ lines. Importantly, such diamonds show very low or null concentration of platelet, with a very weak platelet mode. This result is consistent with the attribution of $D_{b}$ lines to vacancies at interstitial platelets and $D_{a}$ line to some different vacancy variant, probably related to smaller interstitial aggregates. In brief, Fig. 4 is an evidence-based figure of merit which statistically validates the possibility of using the D lines as a new spectroscopic probe of artificial treatment for colour enhancement of Type Ia diamonds with the same reliability of the detection of $\mathrm{H} 1 \mathrm{~b}$ and $\mathrm{H} 1 \mathrm{c}$ centres.

\section{Conclusions}

The analysis of the occurrence of red emission lines belonging to the $\mathrm{D}_{\mathrm{a}}$ and $\mathrm{D}_{\mathrm{b}}$ doublets in a large set of natural Type Ia diamonds - comprising the analysis of a control set of untreated diamonds and a thorough IR characterization of defectiveness in all samples - brings us to assess that the formation mechanism of the vacancy species responsible of the PL signals is related to irradiation followed by thermal treatments which are unlike to occur in nature. Furthermore, the generated light emitting sites - giving rise to the $D_{b}$ lines and/or the room temperature $D_{a}$ line at $681 \mathrm{~nm}$ - turn out to be systematically detected with the H1b and H1c peaks, which in turn are generally considered as a signature of irradiation and heating processes.

It is worth noting that the detailed analysis of both treated and untreated samples gives rise to a strong statistics of a hundred cases in which we register no false positive or false negative, so giving to such an analysis an uncertainty smaller than $1 \%$.

\section{Acknowledgements}

We acknowledge the support and collaboration of the Istituto Gemmologico Italiano (IGI), Italy, under Contract no. 0013885/15. 


\section{Appendix A. Supplementary data}

IR absorption and PL spectra of the investigated natural and treated diamonds.

\section{Reference}

[1] I. Aharonovich, E. Neu, Diamond nanophotonics, Advanced Optical Materials, 2 (2014) 911-928.

[2] R. Balmer, J. Brandon, S. Clewes, H. Dhillon, J. Dodson, I. Friel, P. Inglis, T. Madgwick, M. Markham, T. Mollart, Chemical vapour deposition synthetic diamond: materials, technology and applications, Journal of Physics: Condensed Matter, 21 (2009) 364221.

[3] Y.-R. Chang, H.-Y. Lee, K. Chen, C.-C. Chang, D.-S. Tsai, C.-C. Fu, T.-S. Lim, Y.-K. Tzeng, C.-Y. Fang, C.-C. Han, Mass production and dynamic imaging of fluorescent nanodiamonds, Nature nanotechnology, 3 (2008) 284.

[4] W. Gao, A. Imamoglu, H. Bernien, R. Hanson, Coherent manipulation, measurement and entanglement of individual solid-state spins using optical fields, Nature Photonics, 9 (2015) 363.

[5] N. Mizuochi, T. Makino, H. Kato, D. Takeuchi, M. Ogura, H. Okushi, M. Nothaft, P. Neumann, A. Gali, F. Jelezko, Electrically driven single-photon source at room temperature in diamond, Nature photonics, 6 (2012) 299.

[6] V. Petráková, A. Taylor, I. Kratochvílová, F. Fendrych, J. Vacík, J. Kučka, J. Štursa, P. Cigler, M. Ledvina, A. Fišerová, Luminescence of nanodiamond driven by atomic functionalization: towards novel detection principles, Advanced Functional Materials, 22 (2012) 812-819.

[7] C.G. Yale, F.J. Heremans, B.B. Zhou, A. Auer, G. Burkard, D.D. Awschalom, Optical manipulation of the Berry phase in a solid-state spin qubit, Nature photonics, 10 (2016) nphoton. 2015.2278.

[8] A. Collins, Investigating artificially coloured diamonds, Nature, 273 (1978) 654.

[9] A. Collins, G. Davies, G. Woods, Spectroscopic studies of the H1b and $\mathrm{H} 1 \mathrm{c}$ absorption lines in irradiated, annealed type-la diamonds, Journal of Physics C: Solid State Physics, 19 (1986) 3933.

[10] A.T. Collins, Optical centres produced in diamond by radiation damage, New diamond and frontier carbon technology, 17 (2007) 47-61.

[11] H. Kitawaki, Gem diamonds: Causes of colors, New Diamond and Frontier Carbon Technology, 17 (2007) 119-126.

[12] L. Tretiakova, Spectroscopic methods for the identification of natural yellow gem-quality diamonds, European Journal of Mineralogy, 21 (2009) 43-50.

[13] A.T. Collins, H. Kanda, H. Kitawaki, Colour changes produced in natural brown diamonds by highpressure, high-temperature treatment, Diamond and Related Materials, 9 (2000) 113-122.

[14] A.M. Zaitsev, Optical properties of diamond: a data handbook, Springer Science \& Business Media2013.

[15] I.A. Dobrinets, V.G. Vins, A.M. Zaitsev, HPHT-treated diamonds, Springer2016.

[16] T. Hainschwang, A. Respinger, F. Notari, H.J. Hartmann, C. Günthard, A comparison of diamonds irradiated by high fluence neutrons or electrons, before and after annealing, Diamond and Related Materials, 18 (2009) 1223-1234.

[17] A.T. Collins, The detection of colour-enhanced and synthetic gem diamonds by optical spectroscopy, Diamond and related materials, 12 (2003) 1976-1983.

[18] S.V. Titkov, J.E. Shigley, C.M. Breeding, R.M. Mineeva, N.G. Zudin, A.M. Sergeev, NATURAL-COLOR PURPLE DIAMONDS FROM SIBERIA, Gems \& Gemology, 44 (2008).

[19] G.S. Woods, A.T. Collins, New developments in spectroscopic methods for detecting artificially coloured diamonds, Journal of Gemmology, 20 (1986) 75-83.

[20] A.T. Collins, Colour centres in diamond, Journal of Gemmology, 18 (1982) 27-35.

[21] H. Lim, S. Park, H. Cheong, H.-M. Choi, Y.C. Kim, Discrimination between natural and HPHT-treated type Ila diamonds using photoluminescence spectroscopy, Diamond and Related Materials, 19 (2010) 12541258.

[22] A. Zullino, G. Benedek, A. Paleari, R. Lorenzi, Red emission doublets in diamond from vacancies interacting with interstitial carbon aggregates in tunneling configurations, Carbon, 120 (2017) 294-303. 
[23] B. Dischler, Handbook of Spectral Lines in Diamond: Volume 1: Tables and Interpretations, Springer Science \& Business Media2012.

[24] A. Osvet, A. Yelisseyev, B. Feigelson, N. Mironova, I. Sildos, Optical investigation of Ni impurities in diamond, Radiation effects and defects in solids, 146 (1998) 339-348.

[25] I. Sildos, A. Osvet, Spectral hole burning study of a neutron-irradiated type laB natural diamond, Diamond and Related Materials, 3 (1994) 725-727.

[26] Y. Nisida, Y. Mita, K. Mori, S. Okuda, S. Sato, S. Yazu, M. Nakagawa, M. Okada, Color centers in annealing of neutron-irradiated type Ib and la diamonds, Materials Science Forum, 1989.

[27] V. Vins, A. Eliseev, V. Malogolovets, Optical spectroscopy of synthetic neutron-irradiated diamonds, Sverkhtverdye Materialy, DOI (1988) 18-22.

[28] C.A. Klein, T.M. Hartnett, C.J. Robinson, Critical-point phonon frequencies of diamond, Physical Review B, 45 (1992) 12854.

[29] J. Goss, B. Coomer, R. Jones, C. Fall, P. Briddon, S. Öberg, Extended defects in diamond: the interstitial platelet, Physical review B, 67 (2003) 165208. 


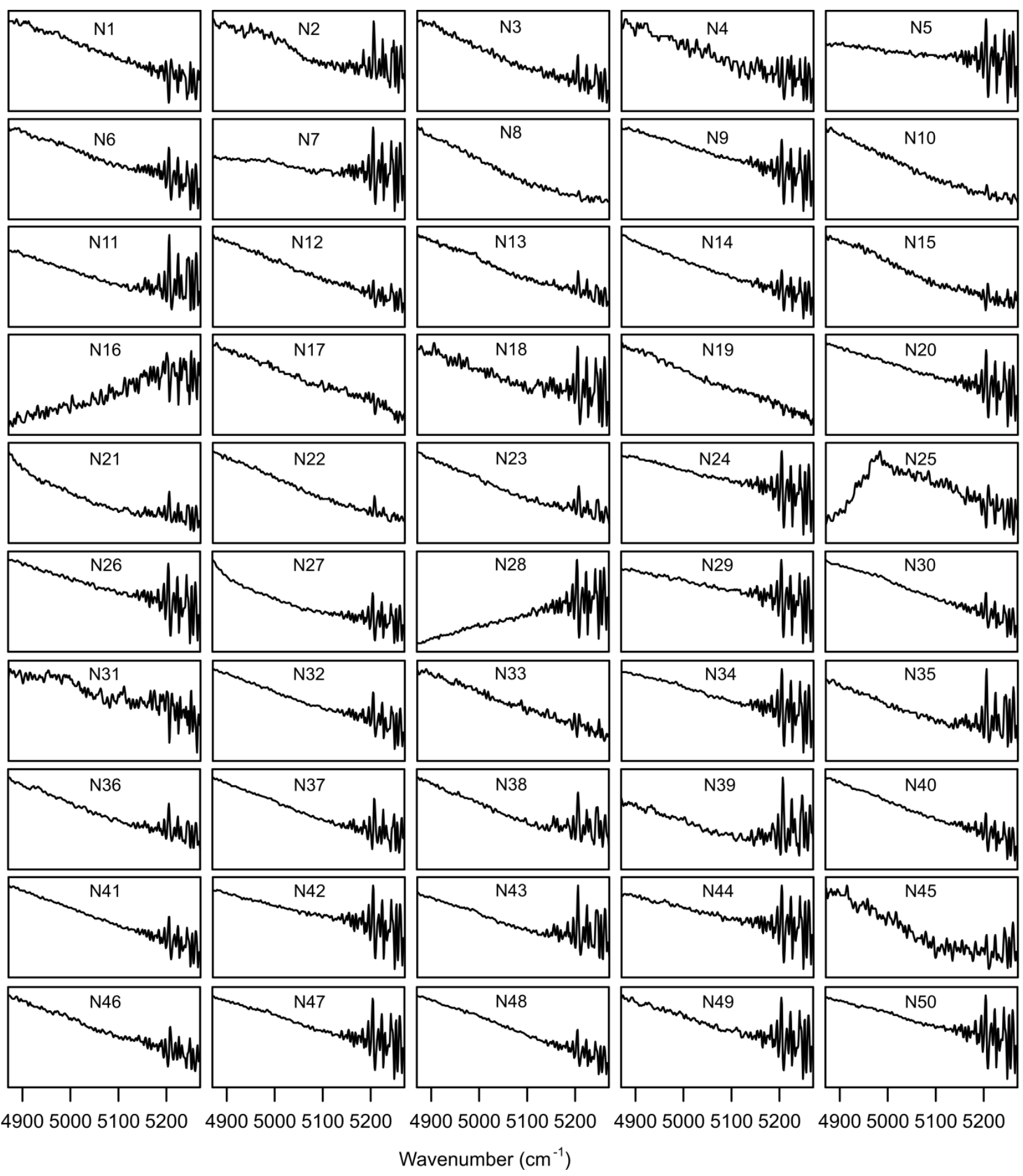

Fig. 1: IR spectrum from DRIFT measurement of certified natural samples, from the investigated set, and normalized in the spectral region $4800-5300 \mathrm{~cm}^{-1}$. 


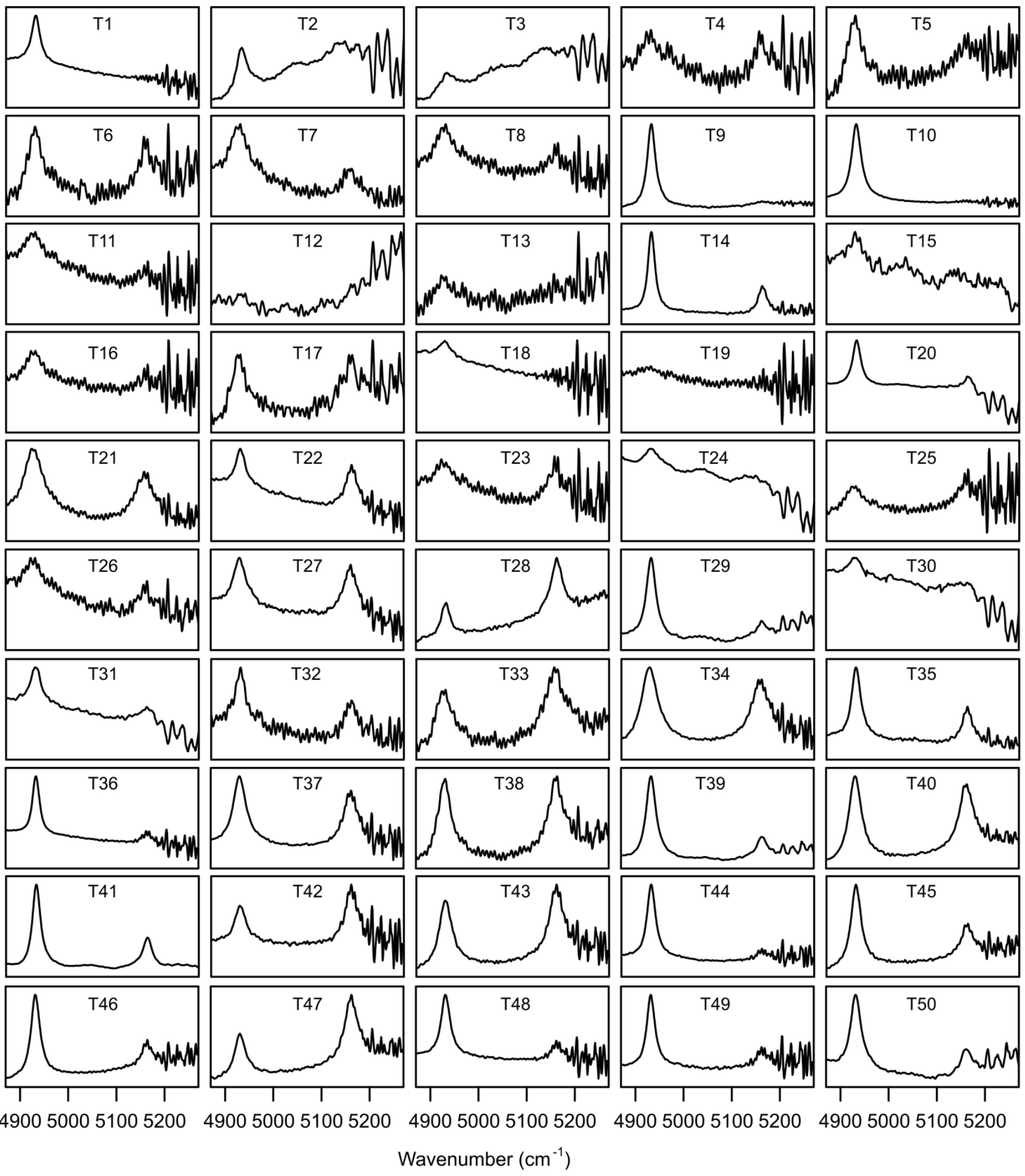

Fig. 2: IR spectrum from DRIFT measurement of treated samples, from the investigated set, and normalized in the spectral region $4800-5300 \mathrm{~cm}^{-1}$. 

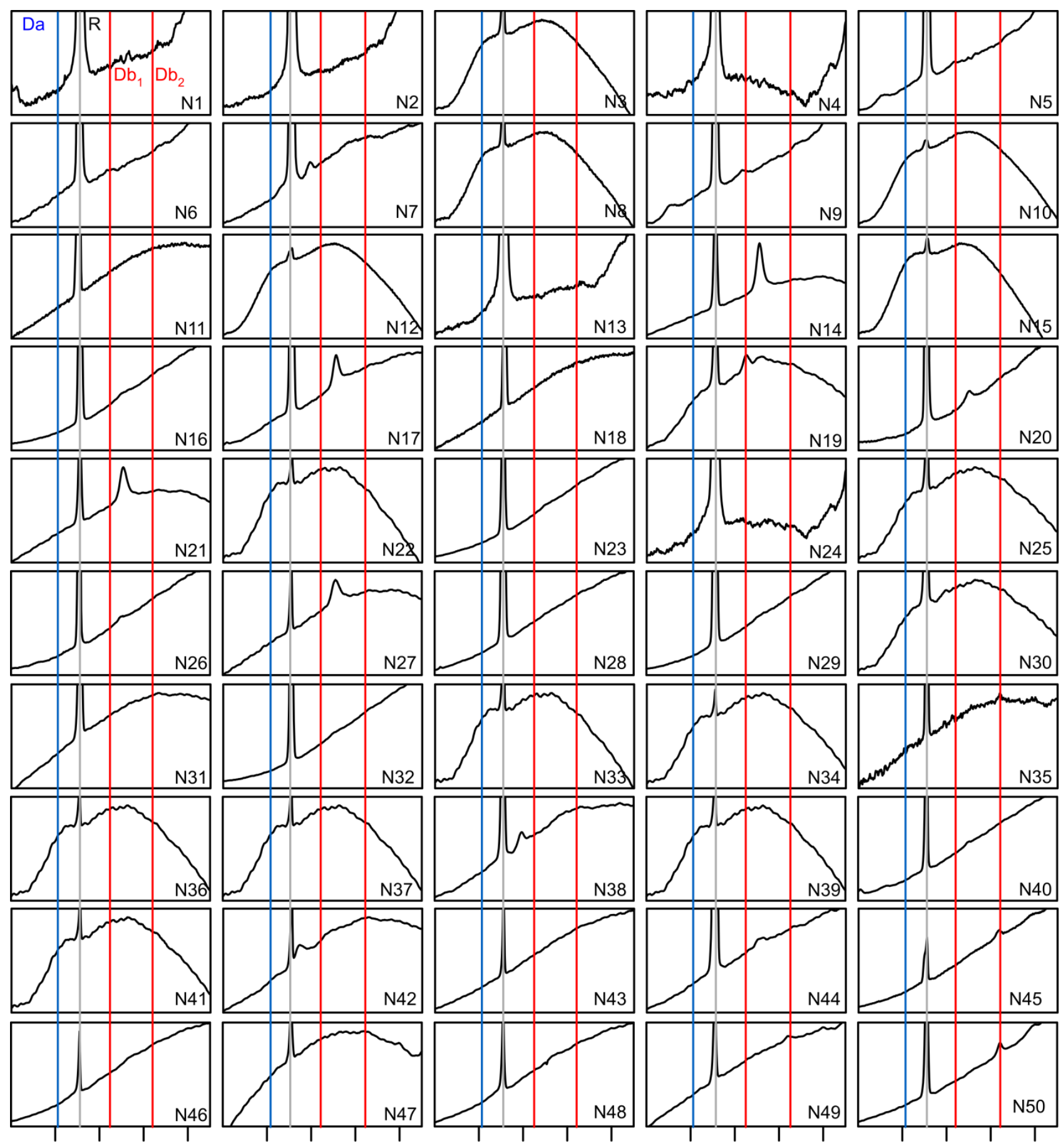

680700720740

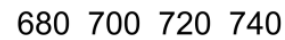

680700720740
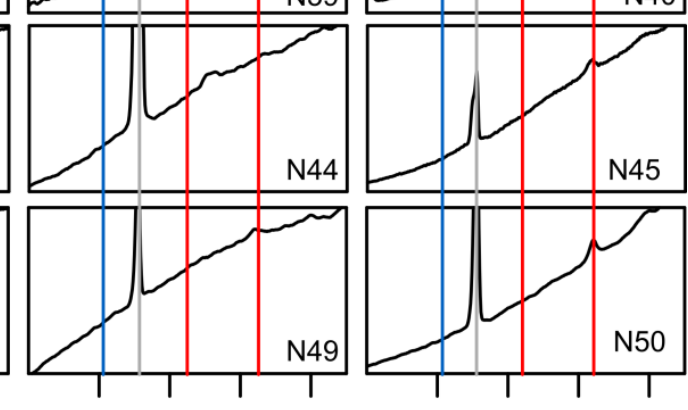

Wavelength (nm)

Fig. 3: PL spectra of the certified natural samples excited at $633 \mathrm{~nm}$. Vertical lines are guides to the eye at the spectral positions of the analysed vacancy light emission lines at 681 (Da), 705 (Db1), and $725 \mathrm{~nm}(\mathrm{Db} 2)$ and Raman line at $691 \mathrm{~nm}(\mathrm{R})$. Spectra are normalized in the spectral range without considering signal from Raman peak. 

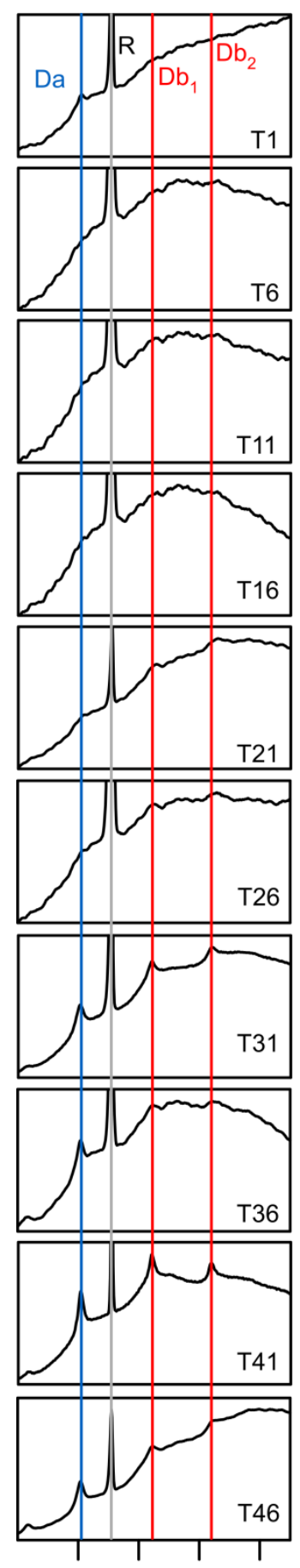

680700720740
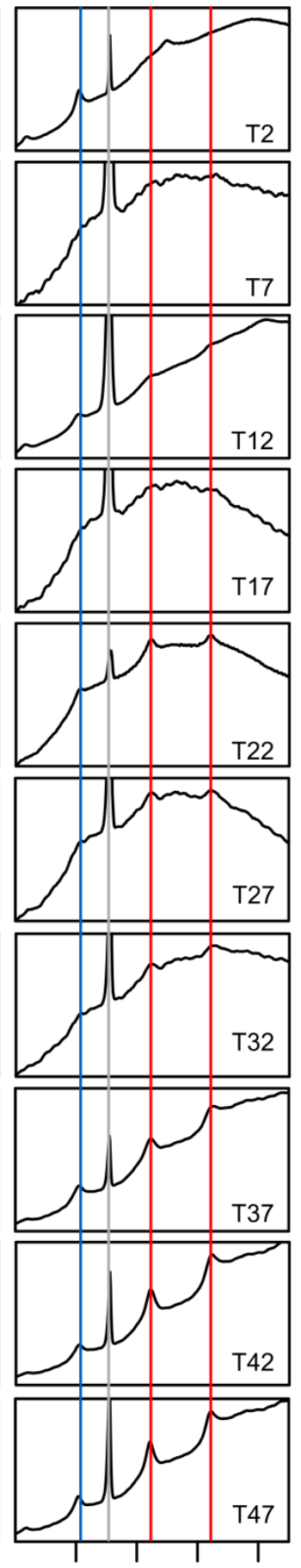

680700720740
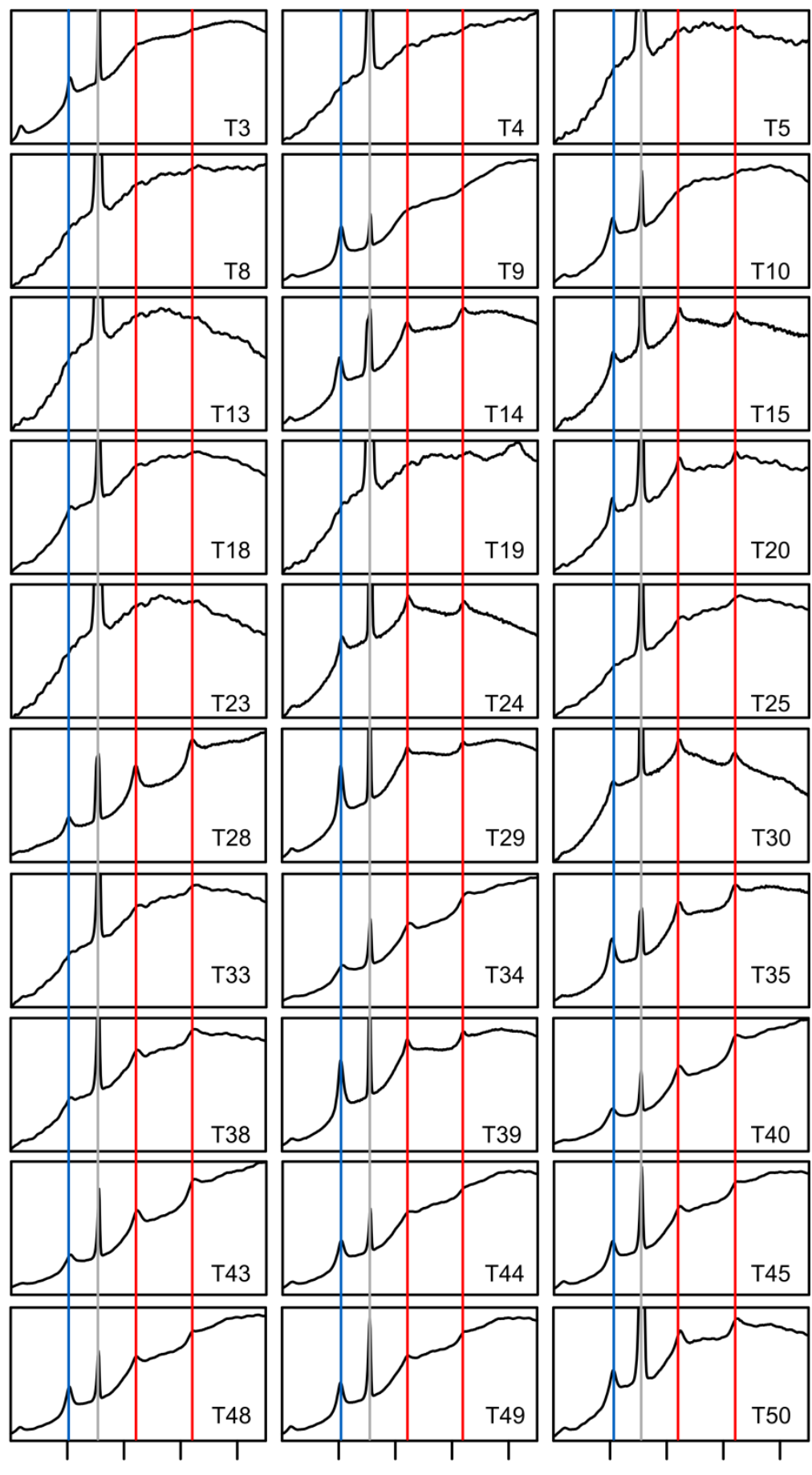

680700720740

Wavelength $(\mathrm{nm})$
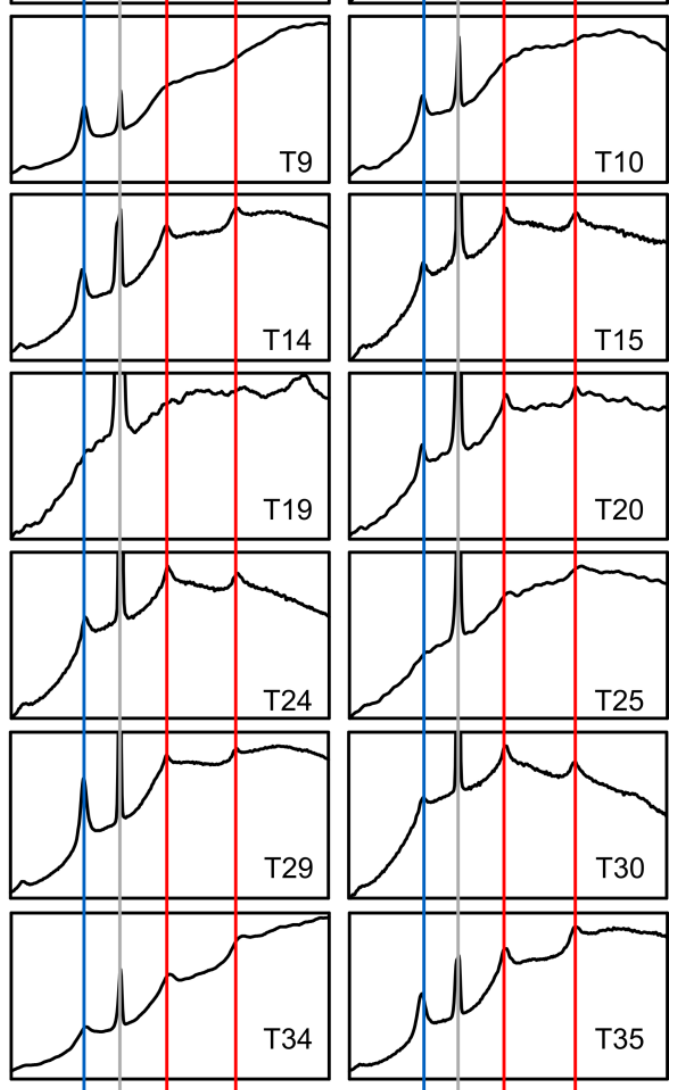

Fig. 4: PL spectra of the treated samples excited at $633 \mathrm{~nm}$. Vertical lines are guides to the eye at the spectral positions of the analysed vacancy light emission lines at 681 (Da), 705 (Db1), and $725 \mathrm{~nm}(\mathrm{Db} 2)$ and Raman line at $691 \mathrm{~nm}(\mathrm{R})$. Spectra are normalized in the spectral range without considering signal from Raman peak. 